\title{
CONCLUDING REMARKS
}

\author{
Tomas Ohlin, Lars Heide, Niels Hoeg, Martti Tienari \\ tomas.ohlin@telo.se,heide.lpf@cbs.dk,nils.hoeg@hydro.com,tienari@cs.Helsinki.fi
}

\begin{abstract}
This is a summary of the First Conference on the History of Nordic Computing. The summary highlights some of the remarks made by panelists and audience participants. Prospects for a second conference on the same theme look promising.
\end{abstract}

Key words: Nordic computing, history, HiNC1

In a final panel session chaired by Tomas Ohlin at the end of the HINC Conference, concluding remarks came from three different perspectives. The participants (mentioned above) commented from the historic perspective, from the industrial perspective and from the academic perspective, with comments stemming from what participants experienced during the conference.

The comments were brief. It became evident that the broad material and the discussions delivered earlier during the conference sessions could have quite different interpretations. This also did not come as a surprise to the participants. On the contrary, people stressed that history is born from descriptions that emerge from different perspectives.

At the end of the discussion, the audience suggested that the Trondheim HINC conference should be the first in a series of conferences and therefore, followed by other Nordic computing conferences. In this discussion, it was mentioned possible organizational updates concerning invitations, refereeing of contributions, and conference marketing. Future organizers should be mindful of conference economics and the possibilities of external support. 
A second HINC conference would naturally concern itself with the development of telecommunications, which the Trondheim HINC did not, since it covered the time up to 1985. A second HINC could also stress historically important applications, education, and information society development to an increased degree. Participants discussed the possible time scope that such a conference would have. Noting the importance of cooperation among all partners with computing history interests, including many US concerns, participants stressed the importance of building active archives, backed by a living website.

The audience was quite active, forming a living dialogue with the panel participants. However, at the time of the Trondheim HINC conference, no plan existed for succeeding conferences. Further organizational efforts would address that issue. 